\title{
A role for LRIG1 in the regulation of malignant glioma aggressiveness
}

\author{
FENG MAO $^{1 *}$, BAOFENG WANG $^{1 *}$, QUNGEN XIAO $^{1}$, GUIFA XI $^{1}$, WEI SUN ${ }^{1}$, HUAQIU ZHANG $^{1}$, \\ FEI YE ${ }^{1}$, FENG WAN ${ }^{1}$, DONGSHENG GUO ${ }^{1}$, TING LEI ${ }^{1}$ and XIAOPING CHEN ${ }^{2}$ \\ Departments of ${ }^{1}$ Neurosurgery and Sino-German Neuro-Oncology Molecular Laboratory and ${ }^{2}$ General Surgery, \\ Tongji Hospital, Tongji Medical College, Huazhong University of Science and Technology, Wuhan 430030, P.R. China
}

Received November 15, 2012; Accepted December 28, 2012

DOI: $10.3892 /$ ijo.2013.1776

\begin{abstract}
The molecular mechanisms that drive the development and aggressive progression of malignant astrocytic tumors remain obscure. Recently, in the search for endogenous negative regulators of EGF receptor, LRIG1 was cloned and characterized as a putative tumor suppressor gene often downregulated in various human tumors, including astrocytic tumors. Although several studies have implicated the function of LRIG1 in the inhibition of tumorigenesis, its precise role and potential underlying mechanisms remain obscure. Therefore, we generated a full-length expression vector to overexpress LRIG1 in the U251 malignant glioma cell line. Introduction of exogenous LRIG1 into glioma cells inhibited cell proliferation manifested by MTT and soft agar clone assay in vitro and subcutaneously tumor xenografts. On the other hand, LRIG1 overexpression inhibited glioma growth by significantly changing the expression pattern of cyclins, resulting in delayed cell cycle. Employing transwell invasion and wound scratch assay and gelatin zymography, LRIG1 inhibited U-251 MG cell invasion and migration by attenuating MMP2 and MMP9 production. Under ligand-stimulated conditions, $\mathrm{p}$-ERK levels did not change, whereas p-AKT levels were inhibited in cells with LRIG1 upregulation, indicating that LRIG1 exerts more inhibiting effects on the PI3K/AKT pathway. Our findings suggest that LRIG1 restricted glioma aggressiveness by inhibiting cell proliferation, migration and invasion. Restoration of LRIG1 to glioma cells could offer a novel therapeutic strategy.
\end{abstract}

Correspondence to: Dr Dongsheng Guo, Department of Neurosurgery and Sino-German Neuro-Oncology Molecular Laboratory, Tongji Hospital, Tongji Medical College, Huazhong University of Science and Technology, 1095 Jiefang Avenue, Wuhan 430030, P.R. China

E-mail: guodongsheng@yahoo.com

${ }^{*}$ Contributed equally

Key words: LRIG1, epidermal growth factor receptor, glioma, proliferation, invasion, migration

\section{Introduction}

Malignant astrocytic tumors are the most common devastating primary neoplasms of the central nervous system with hallmark characteristic of aggressive biological behavior, such as rapid proliferation potential and diffused invasive capability (1). The strong capacity of these tumors to invade and migrate into surrounding normal brain tissue in early stage usually prevents complete removal of the tumor during operation even with radical resection approach and these tumors inevitably recur with no effective treatment (2). Same as in other solid tumors, genetic alterations that result in activation of oncogenes and/or inactivation of tumor suppressor genes are the underlying causes of glioma (3). Sequential gain of oncogenes, such as epidermal growth factor receptor (EGFR) and/or loss of tumor suppressor genes, such as p53, phosphatase and tensin homolog (PTEN), provides the necessary foundation for step-wise progression of malignant glioma from initiation to transformation and aggressive progression $(4,5)$.

Among various identified oncogenes associated with aggressiveness of glioma, EGFR is best characterized. EGFR is amplified in a range of $30-50 \%$ of high-grade malignant astrocytoma cases (6). Enhanced EGFR signaling was proven to contribute to glioma initiation, progression, treatment-resistance and poor survival (7). In a search for negative regulator of EGFR signaling, we had previously cloned and characterized the human leucine-rich repeats and immunoglobulin-like domains 1 (LRIG1) gene (8). LRIG1 belongs to the LRIG gene family, which also includes LRIG2 and LRIG3 (9). LRIG1 is located at chromosome 3p14.3 (10), a region frequently deleted in various human cancers (11). Previous studies demonstrated that LRIG1 was downregulated in conventional renal cell carcinoma, squamous cell carcinoma of the skin and lung (12), breast cancer (13), nasopharyngeal carcinoma (14) and in various human cancer cell lines $(10,15-17)$. LRIG1 negatively regulates EGFR, both by inducing receptor degradation $(18,19)$ and by inhibiting signaling $(20,21)$. Thus, ectopic expression of LRIG1 suppresses glioma cell proliferation $(21,22)$ and invasion (23). However, the exact mechanisms behind these suppressive effects have not been thoroughly analyzed.

In this study, we investigated the effect of LRIG1 overexpression on the malignant glioma cell line U-251 MG and 
analyzed the dynamic cell cycle and screened its potential target cyclins. Next we investigated the effects of LRIG1 on glioma cell invasion and production of MMP2 and MMP9. Moreover, we found that LRIG1 can modulate EGFR downstream signaling pathway-PI3K/AKT expression. Our findings demonstrate LRIG1 may exert its tumor suppressor function via targeting EGFR and its down signaling pathway.

\section{Materials and methods}

Cell culture and transfection. The human glioma cell line U-251 MG purchased from American Type Culture Collection was cultured in Dulbecco's modified Eagle's medium containing $10 \%(\mathrm{v} / \mathrm{v})$ fetal bovine serum (Hyclone, USA) in a humidified $\left(37^{\circ} \mathrm{C}, 5 \% \mathrm{CO}_{2}\right)$ incubator. Cells were transfected with expression vector pLRIG1-GFP, encoding an LRIG1-GFP fusion protein (24) or, as vector control, pEGFP-N1 (Clontech), encoding GFP, using Lipofectamine 2000 (Invitrogen, USA) according to the manufacturer's instructions. G418-resistant clones that represented possible stably transfected cells were ring-cloned and expanded for further experiments.

$R N A$ isolation and $q R T-P C R$. Total RNAs from cultured cells were isolated using TRIzol reagent (Invitrogen) according to the manufacturer's instructions. qRT-PCR was used to evaluate the expression levels of mRNAs as described before (25). Oligonucleotide primer sequences used were as follows: MMP2 sense ATGGATCCTGGCTTTCCC-3' and antisense 5'-GCTTCCAAACTTCACGCTC-3'; MMP9 sense 5'-TGAC AGCGACAAGAAGTG-3' and antisense 5'-CAGTGAAGCGG TACATAGG-3'; LRIG1, 18S primers used were the same as described in a previously published report (16).

Western blot analysis and immunohistochemical staining. Cells were lysed and analyzed by Western blotting as described (26). The following antibodies were used: LRIG1 (Santa Cruz, USA); anti-EGFR, anti-phospho-EGFR (Upstate, USA); antiphospho-ERK, anti-ERK, anti-AKT, anti-phospho-AKT (Cell Signaling, USA); anti-cyclin A, anti-cyclin E, GAPDH (Boster, China); anti-cyclin D1 (Neomarker, USA).

MTT assay and soft agar colony formation assay. The proliferation rate of various cells were measured by 3-[4,5-dimethylthiazol-2-yl]-2,5-diphenyltetrazolium bromide (MTT) cell viability/proliferation assay as described (26). Soft agar colony formation assay was performed as described (27).

Cell cycle synchronization and dynamic cell cycle analysis. Cells grown to $80 \%$ confluency in $10-\mathrm{cm}$ dishes were synchronized in culture medium containing $250 \mathrm{ng} / \mathrm{ml}$ nocodazole for $24 \mathrm{~h}$. Then, detached cells were shaken-off and collected and washed twice with phosphate buffered saline and then, replated subconfluently in complete growth medium. Cells were collected at indicated time-points after nocodazole washout. Dynamic cell cycle were analyzed by flow cytometry using propidium iodide (PI) staining and was performed as previously described (28). Cell lysates were collected at indicated time-points and immunoblotting was performed using anti-cyclin antibodies.
In vitro invasion assay and cell migration assay. In vitro invasion capabilities were measured in transwell chamber assay as previous described (26). Cell migration activities were examined by two-dimensional wound healing assay or in vitro scratch assay as previous described (25).

Gelatin zymography. Serum-free conditioned media were used in SDS-PAGE gelatin-substrate zymography to detect the activity of MMP2 and 9 as described (29). Briefly, 3×10 5 transfected U-251 MG cells were plated in 6-well plates, cultured in $1 \mathrm{ml}$ serum-free DMEM and incubated for $24 \mathrm{~h}$. Twenty microliters of serum-free culture medium per sample were prepared in non-denaturating loading buffer and were sizefractionated in 10\% SDS-polyacrylamide gel impregnated with $0.1 \%$ gelatin. The gels were incubated in a developing buffer for $42 \mathrm{~h}$ at $37^{\circ} \mathrm{C}$. Direct comparisons between separate gels were not made, because the intensity of background staining was variable. Experiments were repeated three times.

In vivo tumor model. Pooled populations of transfected U-251 MG cells were used for subcutaneous tumor growth experiments. Briefly, $1 \times 10^{6}$ glioma cells were injected into both flanks of female BALB/c athymic nude mouse at 4-6 week of age. Tumor volumes were calculated using the following formula: tumor volume $=1 / 2 \mathrm{x}$ (shortest diameter $)^{2} \mathrm{x}$ longest diameter $\left(\mathrm{mm}^{3}\right)$. These experiments were performed in accordance with the guidelines of the Animal Experimental Committee, China Institutes for Biological Sciences.

Statistical analysis. Results are expressed as means \pm standard deviation (SD). Statistical analyses were performed using SPSS statistical software (SPSS Inc., Chicago, IL). Student's t-test was used. Significance was defined as * $\mathrm{p}<0.05$ and ${ }^{* *} \mathrm{p}<0.01$.

\section{Results}

Transformation of U-251 MG cells with LRIG1 expression vector. U-251 MG cells were transfected with pLRIG1-GFP, encoding an LRIG1-GFP fusion protein, or as a vector control, with pEGFP-N1, encoding GFP (green fluorescent protein). Quantitative reverse transcriptase PCR (qRT-PCR) showed that LRIG1 mRNA levels were increased 33-fold in the pLRIG1GFP transformed cells compared to the control cells (Fig. 1A). Western blotting confirmed expression of the LRIG1-GFP fusion protein in the pLRIG1-GFP transformed cells but not in the control cells (Fig. 1B). As previously described $(24,30)$, the LRIG1-GFP fusion protein was mainly located in the plasma membrane (Fig. 1C).

LRIG1 represses cell proliferation by delaying cell cycle progression. Next, we test dynamic cell cycle change in synchronized clonal cell line after exogenous LRIG1 introduction. Most of the control cells left G0/G1 phase and entered S phase at 16-h time-point and entered S phase at 20-h time-point (Fig. 2B, low panel), while most of the LRIG1 overexpression cells started to leave G0/G1 phase and entered $\mathrm{S}$ phase at $20 \mathrm{~h}$ and then, entered $\mathrm{S}$ phase at $24 \mathrm{~h}$ (Fig. 2B, upper panel). This experiment demonstrated that exogenous introduction of LRIG1 into U-251 MG cells caused cell cycle arrest at G0/G1 phase and delayed cell cycle to enter $\mathrm{S}$ phase (Fig. 2A and B). 
A

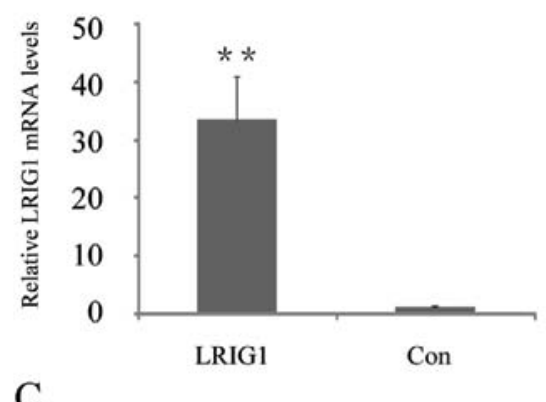

$\mathrm{C}$

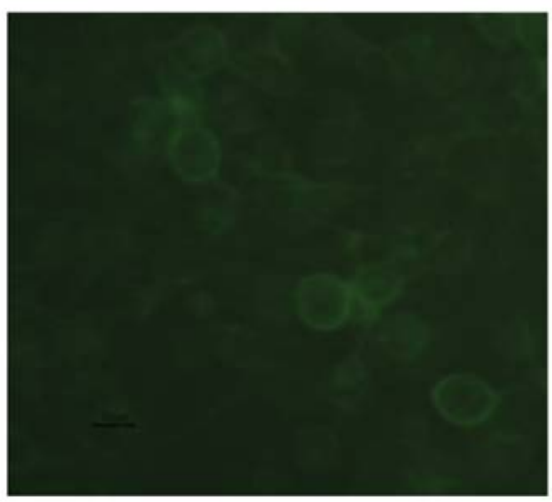

LRIG1
B
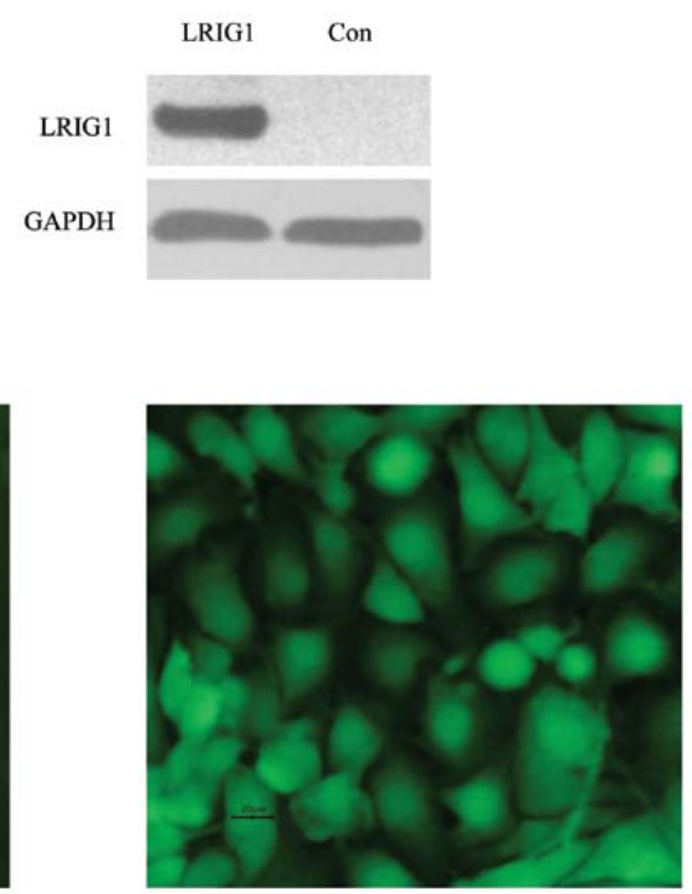

Con

Figure 1. Overexpression of LRIG1 in U-251 MG cells. (A) qRT-PCR analysis and (B) western blot analysis indicated the upregulated expression of LRIG1 in U-251 MG-LRIG1 clone. 18s and GAPDH were used as internal control respectively. (C) Fluorescent microscopy (488 nm) showed stably transfected cells emitted green fluorescence: LRIG1-EGFP cells (left panel); Con-GFP cells (right panel). Scale bars, $20 \mu \mathrm{m}$.

As ectopic expression of LRIG1 in U-251 MG cells delayed cell cycle, we tested whether LRIG1 expression alters the expression of cyclins. As shown in Fig. $2 \mathrm{C}$, the expression of cyclin D1 lasts apparently longer in U-251 MG-LRIG1 cells than control cells, indicating LRIG1 overexpression lengthened the time of the cell cycle. The peak expression level of cyclin E was at 4-h for control group, while it was at 12-16-h in LRIG1 overexpressing cells, indicating the cell cycle was arrested at G0/G1 phase and delayed to enter $\mathrm{S}$ phase. The time course expression pattern of cyclin A was not significantly altered, indicating the entry to $M$ phase was not affected by LRIG1 overexpression. These results were consistent with the dynamic cell cycle analysis shown in Fig. 2A. We next evaluated the effect of LRIG1 on the growth of glioma cells. The results of cell proliferation assay showed that ectopic expression of LRIG1 led to significant inhibition of cell proliferation compared to control cells (Fig. 2D). These results indicate a growth-inhibitory role of LRIG1 on glioma cell line U-251 MG.

LRIG1 inhibits colony formation in vitro and tumorigenicity in vivo. To examine the potential role of LRIG1 in tumorigenesis, the capacity of colony formation was evaluated on LRIG1 overexpressing and control cells. Notably, LRIG1 overexpression cells displayed obviously fewer and smaller colonies compared with control cells (Fig. 3A). This result indicated a growth-inhibitory role of LRIG1 on glioma cells.
To further determine the antitumor effects of LRIG1, LRIG1 overexpression and control cells were injected into five nude mice as described. Although tumor volume between LRIG1 overexpression and control groups was not statistically significant $(\mathrm{p}=0.28$, data not shown), there was a trend for loss of LRIG1 with a higher risk with increasing tumor size. The average tumor volume was $106.4 \mathrm{~mm}^{3}$ in control group vs $6.89 \mathrm{~mm}^{3}$ in LRIG1 overexpression group at the end of eighth week after implantation. The tumor incidence was $80 \%$ (8 tumors/10 injection sites) in control group vs $30 \%$ (3 tumors/10 injection sites) in LRIG1 overexpression group (Fig. 3B and C). The results indicate that overexpression of LRIG1 inhibited tumorigenicity of U-251 MG cells in the nude mouse xenograft model.

LRIG1 inhibits U-251 MG cell migration and invasion and decreases secretion and activity of MMP2 and MMP9. In the following part of this study, we investigated whether overexpression of LRIG1 could affect cell invasion and migration. We found that invasion cells in the LRIG1 overexpressing cells were decreased to $12 \%$ of that of the control cells (Fig. 4A). Cell migration capability was further tested by wound scratch assay. The relative migrated distances of LRIG1 overexpressing cells were $45 \%$ of the distances migrated by the control cells at $48 \mathrm{~h}$ (Fig. 4B).

Gelatin zymography assay was performed to investigate the effect of the LRIG1 on the activity of MMP2 and MMP9. 
A
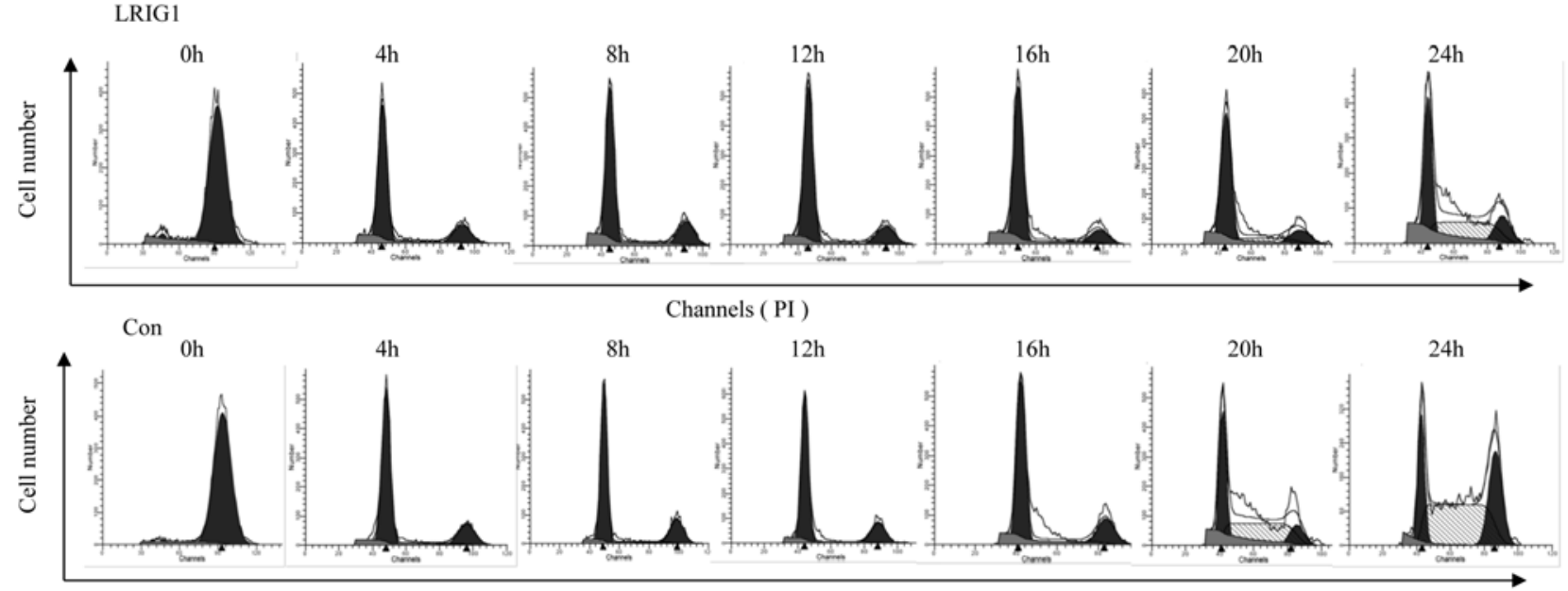

Channels ( PI )

B

\begin{tabular}{|cccccccc|}
\hline LRIG1 & $0 \mathrm{~h}$ & $4 \mathrm{~h}$ & $8 \mathrm{~h}$ & $12 \mathrm{~h}$ & $16 \mathrm{~h}$ & $20 \mathrm{~h}$ & $24 \mathrm{~h}$ \\
\hline $\mathrm{G} 0 / \mathrm{G} 1(\%)$ & 3.61 & 75.78 & 73.29 & 76.74 & 76.35 & 66.79 & 41.67 \\
$\mathrm{~S}(\%)$ & 0.03 & 4.6 & 4.28 & 5.55 & 9.76 & 18.76 & 43.16 \\
$\mathrm{G} 2 / \mathrm{M}(\%)$ & 96.35 & 19.62 & 22.43 & 17.72 & 13.89 & 14.45 & 15.17 \\
\hline
\end{tabular}

\begin{tabular}{|cccccccc|}
\hline Con & 0h & $4 \mathrm{~h}$ & $8 \mathrm{~h}$ & $12 \mathrm{~h}$ & $16 \mathrm{~h}$ & $20 \mathrm{~h}$ & $24 \mathrm{~h}$ \\
\hline G0/G1(\%) & 0.98 & 73.75 & 73.27 & 76.84 & 68.75 & 37.59 & 19.25 \\
$\mathrm{~S}(\%)$ & 0.87 & 6.15 & 4.81 & 2.37 & 9.66 & 51.13 & 53.43 \\
G2/M(\%) & 98.15 & 20.1 & 21.92 & 20.79 & 21.6 & 11.28 & 27.33 \\
\hline
\end{tabular}

$\mathrm{D}$

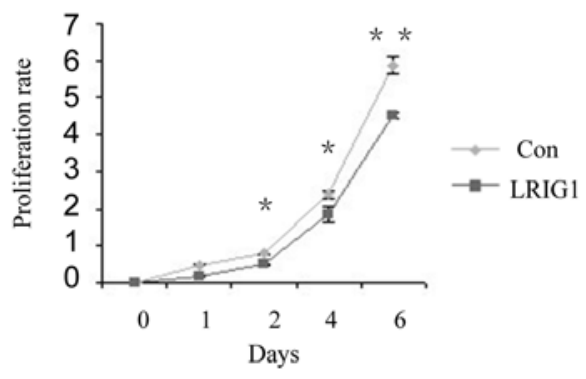

$\mathrm{C}$ Con $\quad$ G2/M release (nocodazole washout) \begin{tabular}{lllllllll}
\multicolumn{2}{c}{ LRIG1 } & \multicolumn{7}{l}{ G2/M release (nocodazole washout) } \\
\hline 0.5 & 2 & 4 & 6 & 8 & 12 & 16 & 20 & $24(\mathrm{~h})$
\end{tabular}
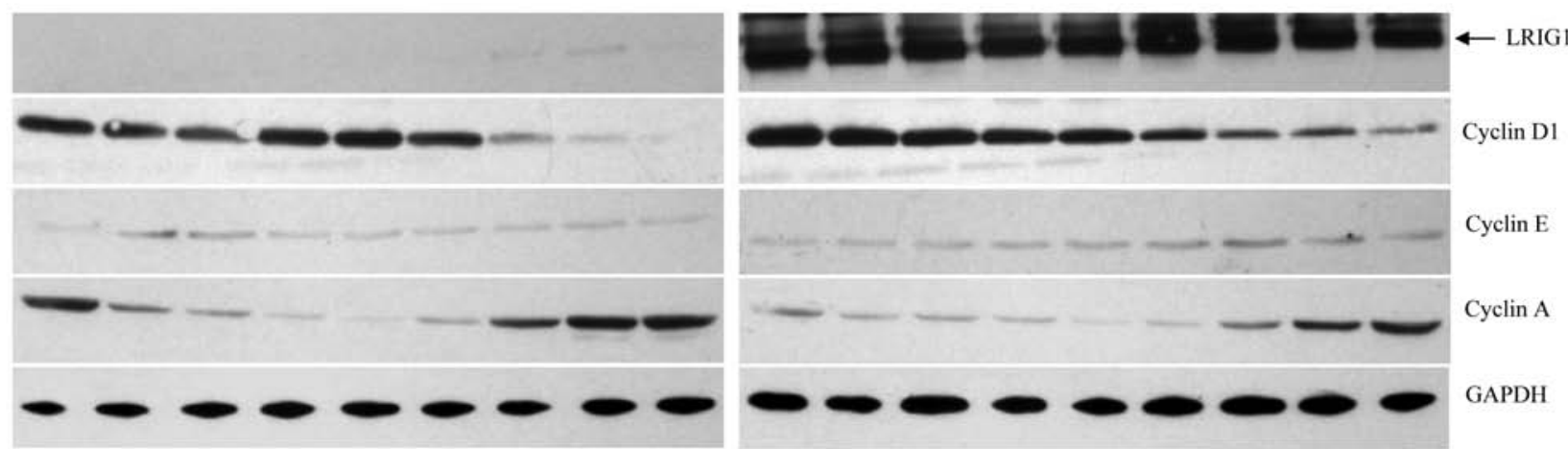

Figure 2. LRIG1 delays cell cycle progression and inhibits growth in U-251 MG cells. (A) Flow cytometry analysis of propidium iodide-stained cells for each of the indicated time-points showed dynamic cell cycle distribution. Control cells and the LRIG1 overexpressing cells were blocked in G2/M by incubation with the microtubule inhibitor nocodazole. Then, the synchronized cells were released from the cell cycle block by washing out the inhibitor. (B) Time course of cell cycle distribution. (C) Deregulation of cyclin D1, E and A expression in stable LRIG1 and Con transfected U-251 MG cells. Cell lysates were collected at different time-points after nocodazole washout and immunobloting was performed using anti-cyclin D1, A and E antibodies. (D) Growth curves were measured by MTT assay for the times as indicated. The data are presented as mean \pm SD of pentaplicate wells in one experiment and representative of three independent experiments. ${ }^{*} \mathrm{p}<0.05 ;{ }^{* *} \mathrm{p}<0.01$.

After exogenous introduction of LRIG1 into U-251 MG cells, MMP9 activity was significantly decreased, while MMP2 activity was modestly decreased (Fig. 4C). To further confirm the effect of LRIG1 on MMPs, quantitative real-time PCR results showed that after exogenous introduction of LRIG1 in to U-251 MG cell, the MMP2 mRNA levels were decreased to $44.8 \%$ and MMP9 mRNA levels were decreased to $39.5 \%$, compared to control group (Fig. 4D). These results suggest that 
A

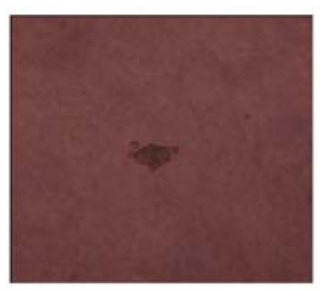

LRIG1

B

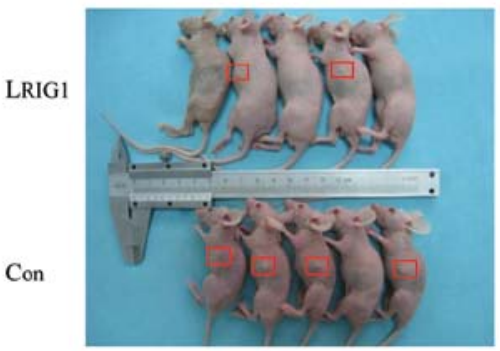

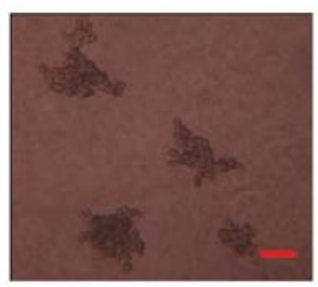

Con

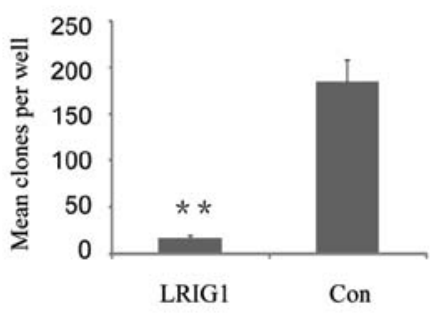

$\mathrm{C}$

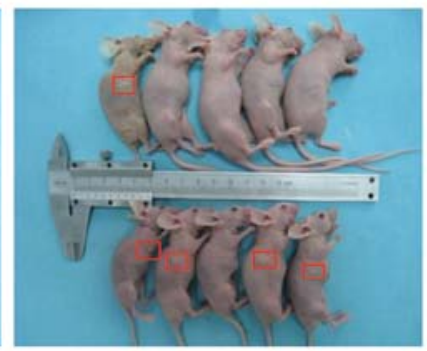

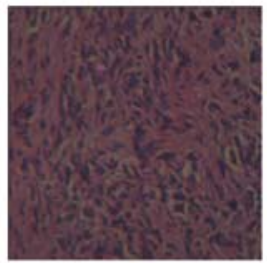

LRIG1

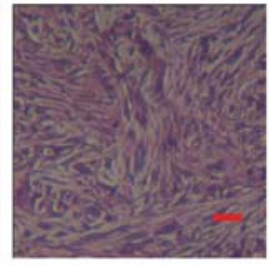

Con

Figure 3. Effects of LRIG1 on U-251 MG growth in vitro and tumorigenicity in vivo. (A) Left panel shows representative images of colony formation in soft agar. Right panel shows quantification of colony formation. Scale bar, $25 \mu \mathrm{m}$. The data are presented as the means \pm SD of triplicate wells. ${ }^{* *}$ p $<0.01$. (B) Images of Con-U-251 MG and LRIG1-U-251 MG subcutaneous tumors 8 weeks after inoculation. Tumor sites are marked by red frames. (C) Tumor sections were fixed in $4 \%$ paraformaldehyde and stained with $\mathrm{H} \& \mathrm{E}$ to determine histologic morphology.

A

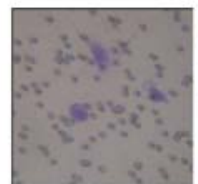

LRIG1

\section{B}

$0 \mathrm{~h}$

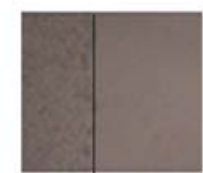

$48 \mathrm{~h}$

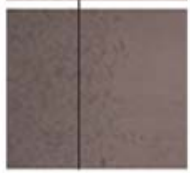

LRIGI

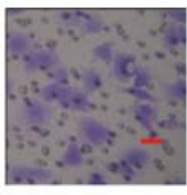

Con
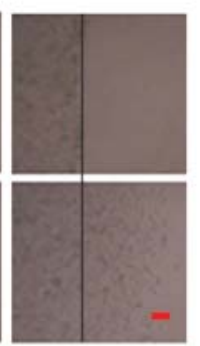

Con
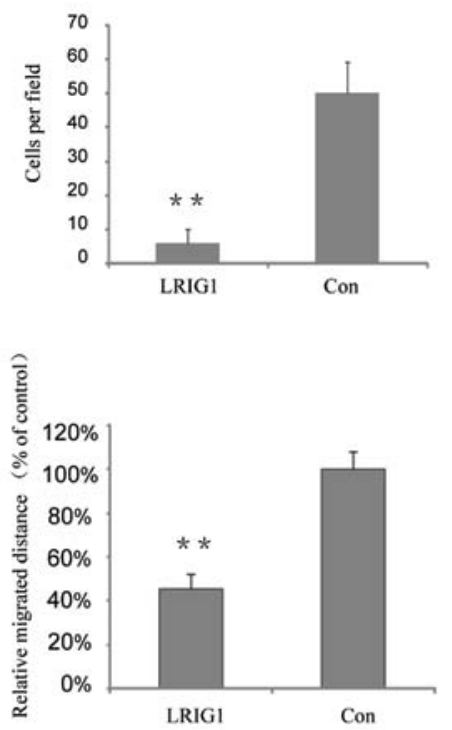

C

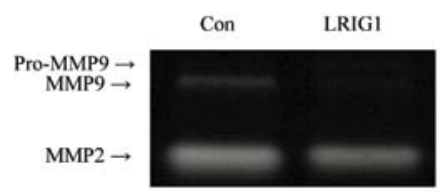

D

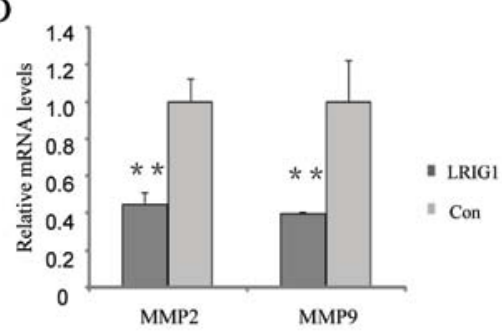

Figure 4. LRIG1 inhibits U-251 MG cell migration and invasion by decreasing secretion and activity of MMP2 and MMP9. (A) The invasion ability was measured by the transwell invasion chamber assay as overexpression of LRIG1 in U-251 MG cells. Scale bar, $25 \mu \mathrm{m}$. The numbers of the invaded cells were counted in five fields of vision of the filter and the results from three separate chambers were then numerically averaged. The data are presented as the means \pm SD of triplicate wells. ${ }^{* *}$ p $<0.01$. (B) Two-dimensional migration assays were conducted with a wound healing assay. The migration distance during the designated period was measured. Scale bar, $100 \mu \mathrm{m}$. The data are presented as the means \pm SD of triplicate wells. ${ }^{* *}$ p $<0.01$. (C) Samples of conditioned medium were assayed for MMP2 and MMP9 by gelatin zymography. (D) mRNA levels of MMP2, MMP9 were quantified by real-time RT-PCR, 18s was used as internal control. The data are presented as mean $\pm \mathrm{SD}$ and representative of three independent experiments. ${ }^{* * *} \mathrm{p}<0.01$. 


$\begin{array}{rcccccc}\text { EGF(min) } & 0 & 0 & 5 & 5 & 30 & 30 \\ \text { Con } & \text { LRIG1 } & \text { Con } & \text { LRIG1 } & \text { Con } & \text { LRIG1 }\end{array}$

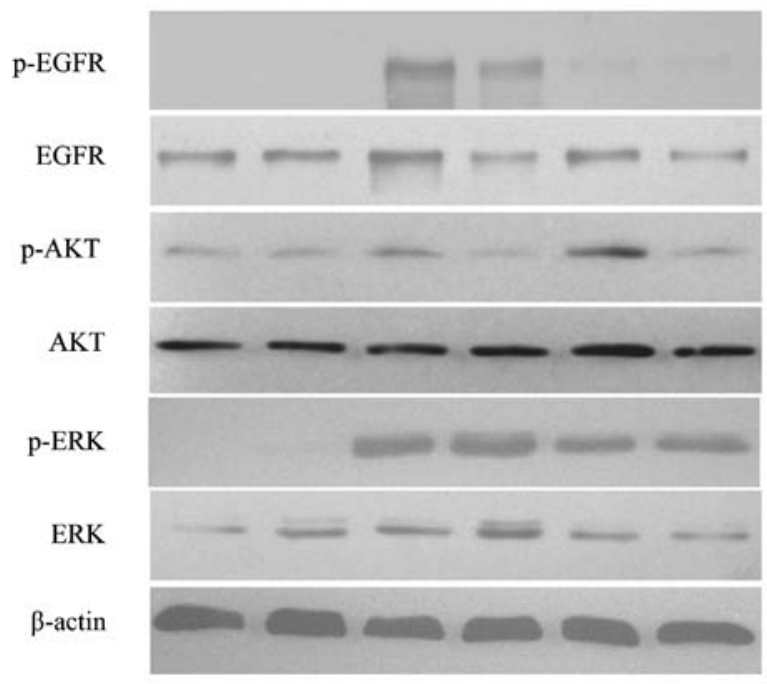

Figure 5. LRIG1 inhibits EGF signaling and its downstream-PI3K/AKT pathway in U251 MG cells. Cells were starved for $24 \mathrm{~h}$, then stimulated in the presence of EGF $(100 \mathrm{ng} / \mathrm{ml})$ for the indicated time. Cell lysates were analyzed using the indicated antibodies. The Western blots shown are representive of three independent experiments.

LRIG1 plays an important role in suppressing MMP2 and 9 production and is involved in invasion and migration in U-251 MG cells.

LRIG1 exerts more effect on PI3K/AKT pathway than MAPK/ ERK1/2 pathway. In the next experiments, we confirmed that LRIG1 negatively regulated the levels of EGFR and phosphorylated EGFR in our experimental system (Fig. 5) (19). We then analyzed the expressions and phosphorylation of two key intracellular signal molecules, AKT and ERK1/2. The basal expression levels of AKT and ERK were not altered. Further, we showed the levels of phosphorylated AKT decreased in LRIG1 overexpressing cells, while the phosphorylated ERK level was not altered, indicating the LRIG1 exerts more influence on intracellular PI3K/AKT pathway than MAPK/ERK1/2 pathway (Fig. 5).

\section{Discussion}

In this study, we provided that LRIG1 functioned as a negative growth regulator for glioma cells and showed that overexpression of LRIG1 inhibited glioma to form clonies in vitro and to develop tumors in vivo. Ectopic expression of LRIG1 suppressed the invasion and migration of cultured glioma cells by regulating secreation of MMP2 and MMP9. These observations provide further understanding of the molecular mechanisms of inhibitory effects of LRIG1 on glioma cell aggressiveness.

Malignant astrocytic tumors are the most aggressive and highly lethal type of brain tumor with a strong ability of rapid proliferation and diffuse invasion into surrounding normal brain tissue. While the molecular events involved in the initiation of glioblastoma are not fully understood, it is believed that, deregulated tumor cell proliferation appears to be a critical early event in glioma development. To assess the potential role of LRIG1 in glioma cell growth, we overexpressed LRIG1 in glioma cell line U-251 MG. Our study revealed that LRIG1 overexpression resulted in decreased tumor cell growth confirmed by MTT and soft agar colony assay. We further showed that LRIG1 overexpression inhibits glioma cell proliferation in vivo. Cellular proliferation is tightly regulated by progression through the cell cycle of DNA synthesis and mitosis by the formation and activity of cyclin-cyclin-dependent-kinase (CDK) complexes (31). We demonstrated that enhanced expression of LRIG1 in glioma cells significantly changed cell cycle control protein cyclin D1, E expression pattern, resulting in cell cycle delay at G0/G1. The strong exogenous LRIG1 regulation over cell cycle control further implies that fundamental alterations of cyclins and tumor suppressors result in deregulated cell cycle and unrestrained cell proliferation (32).

In addition to its function as a potent negative growth regulator, the present and our previous study showed that LRIG1 overexpression led to a significant decrease of glioblastoma cell migration and invasion (23), while the molecular mechanisms of its inhibitory effects on glioma cell metastasis still remain obscure. Matrix metalloproteinases (MMPs) are a family of extracellular zinc-dependent endopeptidases that selectively cleave the protein components of the extracellular matrix (33). Numerous studies have demonstrated that malignant glioma cells secrete MMPs to facilitate their migration and invasion (34). We found that overexpression of LRIG1 inhibited glioma cell invasion and migration by suppressing MMP2 and 9 production. Because EGFR activation can promote MMP2 (35) and MMP9 expression $(36,37)$ and LRIG1 negatively regulated the level of EGFR, we deduced that LRIG1 regulated glioma cell metastasis by attenuating EGFR signaling pathway, at least in part.

Accumulating evidence has identified the EGFR and its downstream signal networks as commonly deregulated components in the oncogenesis of glioma, especially primary GBM $(1,38)$. Once activated and subsequent dimerization, EGFR activates a variety of intracellular phosphorylationmediated signal transduction events (38). The important events in glioma biology include the RAS/mitogen activated protein kinase (MAPK) and phosphatidylinositol 3-kinase (PI3K)/ AKT pathways that regulate cell proliferation, survival and invasion $(1,38)$. Upregulation of LRIG1 influenced expression levels of p-AKT rather than p-ERK in response to ligand stimulation. Constitutive activation of PI3K/AKT signaling pathway plays a pivotal role in the pathogenesis and malignant progression of several human cancers, including glioblastoma (39). Herein, we found that overexpression of LRIG1 negatively regulated glioma cell proliferation, migration and invasion, possibly by inhibiting EGFR activation and its downstream signaling PI3K/AKT pathway.

Collectively, we identified that LRIG1 overexpression resulted in decreased cell growth in vitro and in vivo and delay in cell cycle by changing the expression pattern of cyclins. LRIG1 also attenuated invasion of glioma cells by decreasing production of MMP2 and 9. Analysis of the signaling pathways revealed that LRIG1 regulated EGFR signaling pathway transduction involved in the regulation of tumour cell prolife- 
ration and migration and invasion. It was demonstrated that LRIG1 exerts its tumor suppressor function and implicates its potential targets for future brain tumor treatments.

\section{Acknowledgements}

This study was supported by National Natural and Science Foundation of China (No. 81001116).

\section{References}

1. Furnari FB, Fenton T, Bachoo RM, et al: Malignant astrocytic glioma: genetics, biology and paths to treatment. Genes Dev 21: 2683-2710, 2007.

2. MacDonald TJ, Aguilera D and Kramm CM: Treatment of highgrade glioma in children and adolescents. Neurooncology 13 1049-1058, 2011.

3. Sathornsumetee S, Reardon DA, Desjardins A, Quinn JA, Vredenburgh JJ and Rich JN: Molecularly targeted therapy for malignant glioma. Cancer 110: 13-24, 2007.

4. Smith JS, Tachibana I, Passe SM, et al: PTEN mutation, EGFR amplification and outcome in patients with anaplastic astrocytoma and glioblastoma multiforme. J Natl Cancer Inst 93: 1246-1256, 2001.

5. Reifenberger G and Collins VP: Pathology and molecular genetics of astrocytic gliomas. J Mol Med 82: 656-670, 2004.

6. Frederick L, Wang XY, Eley G and James CD: Diversity and frequency of epidermal growth factor receptor mutations in human glioblastomas. Cancer Res 60: 1383-1387, 2000.

7. Heimberger AB, Hlatky R, Suki D, et al: Prognostic effect of epidermal growth factor receptor and EGFRvIII in glioblastoma multiforme patients. Clin Cancer Res 11: 1462-1466, 2005.

8. Nilsson J, Vallbo C, Guo D, et al: Cloning, characterization and expression of human LIG1. Biochem Biophys Res Commun 284 $1155-1161,2001$.

9. Guo D, Holmlund C, Henriksson R and Hedman H: The LRIG gene family has three vertebrate paralogs widely expressed in human and mouse tissues and a homolog in Ascidiacea. Genomics 84: 157-165, 2004.

10. Hedman H, Nilsson J, Guo D and Henriksson R: Is LRIG1 a tumour suppressor gene at chromosome 3p14.3? Acta Oncol 41: $352-354,2002$

11. Knuutila S, Aalto Y, Autio K, et al: DNA copy number losses in human neoplasms. Am J Pathol 155: 683-694, 1999.

12. Boelens MC, van den Berg A, Fehrmann RS, et al: Current smoking-specific gene expression signature in normal bronchial epithelium is enhanced in squamous cell lung cancer. J Pathol 218: 182-191, 2009.

13. Miller JK, Shattuck DL, Ingalla EQ, et al: Suppression of the negative regulator LRIG1 contributes to ErbB2 overexpression in breast cancer. Cancer Res 68: 8286-8294, 2008.

14. Sheu JJ, Lee CH, Ko JY, et al: Chromosome 3p12.3-p14.2 and 3q26.2-q26.32 are genomic markers for prognosis of advanced nasopharyngeal carcinoma. Cancer Epidemiol Biomarkers Prev 18: 2709-2716, 2009.

15. Tanemura A, Nagasawa T, Inui S and Itami S: LRIG-1 provides a novel prognostic predictor in squamous cell carcinoma of the skin: immunohistochemical analysis for 38 cases. Dermatol Surg 31: 423-430, 2005

16. Thomasson M, Hedman H, Guo D, Ljungberg B and Henriksson R: LRIG1 and epidermal growth factor receptor in renal cell carcinoma: a quantitative RT-PCR and immunohistochemical analysis. Br J Cancer 89: 1285-1289, 2003.

17. Yang WM, Yan ZJ, Ye ZQ and Guo DS: LRIG1, a candidate tumour-suppressor gene in human bladder cancer cell line BIU87. BJU Int 98: 898-902, 2006.

18. Gur G, Rubin C, Katz M, et al: LRIG1 restricts growth factor signaling by enhancing receptor ubiquitylation and degradation. EMBO J 23: 3270-3281, 2004.
19. Laederich MB, Funes-Duran M, Yen L, et al: The leucine-rich repeat protein LRIG1 is a negative regulator of ErbB family receptor tyrosine kinases. J Biol Chem 279: 47050-47056, 2004.

20. Goldoni S, Iozzo RA, Kay P, et al: A soluble ectodomain of LRIG1 inhibits cancer cell growth by attenuating basal and ligand-dependent EGFR activity. Oncogene 26: 368-381, 2007.

21. Yi W, Holmlund C, Nilsson J, et al: Paracrine regulation of growth factor signaling by shed leucine-rich repeats and immunoglobulin-like domains 1. Exp Cell Res 317: 504-512, 2011.

22. Stutz MA, Shattuck DL, Laederich MB, Carraway KL III and Sweeney C: LRIG1 negatively regulates the oncogenic EGF receptor mutant EGFRvIII. Oncogene 27: 5741-5752, 2008.

23. Ye F, Gao Q, Xu T, et al: Upregulation of LRIG1 suppresses malignant glioma cell growth by attenuating EGFR activity. J Neurooncol 94: 183-194, 2009.

24. Nilsson J, Starefeldt A, Henriksson R and Hedman H: LRIG1 protein in human cells and tissues. Cell Tissue Res 312: 65-71, 2003.

25. Song H, Li Y, Lee J, Schwartz AL and Bu G: Low-density lipoprotein receptor-related protein 1 promotes cancer cell migration and invasion by inducing the expression of matrix metalloproteinases 2 and 9. Cancer Res 69: 879-886, 2009.

26. Wang B, Han L, Chen R, et al: Downregulation of LRIG2 expression by RNA interference inhibits glioblastoma cell (GL15) growth, causes cell cycle redistribution, increases cell apoptosis and enhances cell adhesion and invasion in vitro. Cancer Biol Ther 8: 1018-1023, 2009.

27. Lau YK, Murray LB, Houshmandi SS, Xu Y, Gutmann DH and $\mathrm{Yu}$ Q: Merlin is a potent inhibitor of glioma growth. Cancer Res 68: 5733-5742, 2008

28. Cai M, Han L, Chen R, et al: Inhibition of LRIG3 gene expression via RNA interference modulates the proliferation, cell cycle, cell apoptosis, adhesion and invasion of glioblastoma cell (GL15). Cancer Lett 278: 104-112, 2009.

29. Galis ZS, Muszynski M, Sukhova GK, et al: Cytokine-stimulated human vascular smooth muscle cells synthesize a complement of enzymes required for extracellular matrix digestion. Circ Res 75: 181-189, 1994.

30. Guo D, Nilsson J, Haapasalo H, et al: Perinuclear leucine-rich repeats and immunoglobulin-like domain proteins (LRIG1-3) as prognostic indicators in astrocytic tumors. Acta Neuropathol 111: 238-246, 2006.

31. Malumbres $M$ and Barbacid M: Cell cycle, CDKs and cancer: a changing paradigm. Nat Rev Cancer 9: 153-166, 2009.

32. Vermeulen K, Van Bockstaele DR and Berneman ZN: The cell cycle: a review of regulation, deregulation and therapeutic targets in cancer. Cell Prolif 36: 131-149, 2003.

33. Stamenkovic I: Extracellular matrix remodelling: the role of matrix metalloproteinases. J Pathol 200: 448-464, 2003.

34. Nakada M, Okada Y and Yamashita J: The role of matrix metalloproteinases in glioma invasion. Front Biosci 8: e261-269, 2003.

35. Kodali R, Hajjou M, Berman AB, et al: Chemokines induce matrix metalloproteinase- 2 through activation of epidermal grow th factor receptor in arterial smooth muscle cells. Cardiovasc Res 69: 706-715, 2006.

36. Alper O, Bergmann-Leitner ES, Bennett TA, Hacker NF, Stromberg K and Stetler-Stevenson WG: Epidermal growth factor receptor signaling and the invasive phenotype of ovarian carcinoma cells. J Natl Cancer Inst 93: 1375-1384, 2001

37. Cox G, Jones JL and O'Byrne KJ: Matrix metalloproteinase 9 and the epidermal growth factor signal pathway in operable non-small cell lung cancer. Clin Cancer Res 6: 2349-2355, 2000.

38. Huang PH, Xu AM and White FM: Oncogenic EGFR signaling networks in glioma. Sci Signal 2: re6, 2009.

39. Knobbe CB, Trampe-Kieslich A and Reifenberger G: Genetic alteration and expression of the phosphoinositol-3-kinase/Akt pathway genes PIK3CA and PIKE in human glioblastomas. Neuropathol Appl Neurobiol 31: 486-490, 2005. 